\title{
Care of Mother and Neonate during COVID- 19 Pandemic for a Healthy Future
}

\author{
Poonam Joshi', L Levis Murry', S Satchala Dinakaran ${ }^{3}$ \\ ${ }^{1,2}$ Associate Professor, College of Nursing, AlIMS, New Delhi, India. \\ ${ }^{3}$ Assistant Secretary General, L 17, 18 Trained Nurses Association of India Green Park, New Delhi, India. \\ DOI: https://doi.org/10.24321/2455.9318.202014
}

\section{I $\quad \mathbf{N} \quad \mathbf{F} \quad \mathbf{O}$}

\author{
Corresponding Author: \\ Poonam Joshi, College of Nursing, AlIMS, New \\ Delhi, India. \\ E-mail Id: \\ pjoshi495@gmail.com \\ Orcid Id: \\ https://orcid.org/0000-0002-7016-8437 \\ How to cite this article: \\ Joshi P, Murry LL, Dinakaran SV. Care of Mother \\ and Neonate during COVID-19 Pandemic for \\ a Healthy Future. Int J Nurs Midwif Res 2020; \\ 7(2): 21-26. \\ Date of Submission: 2020-06-19 \\ Date of Acceptance: 2020-10-27
}

\section{$\begin{array}{llllllllllll}\mathbf{A} & \mathbf{B} & \mathbf{S} & \mathbf{T} & \mathbf{R} & \mathbf{A} & \mathbf{C} & \mathbf{T}\end{array}$}

COVID-19 caused by coronavirus 2 (SARS-CoV-2) was first time reported in December 2019. The disease has been showing rapid progression to a global pandemic causing profound adverse impact on medical, social, and economic status of the world. Pregnant women and new-borns belong to vulnerable population, who need to be protected. However, currently the impact of this novel virus on the foetus and neonate remains unknown. This article outlines the precautions and steps to be taken by nurses, an important member of the Health Care Team (HCT) before, and during delivery, during and after resuscitation of a neonate born to a COVID-19 mother. More evidence and research work are needed to know the risk of vertical and horizontal transmission of SARS-CoV-2 and its impact on maternal, fetal and neonatal outcomes.

Keywords: Neonatal Resuscitation, COVID-19, SARS-CoV-2, Pregnant Women, Neonates

\section{Introduction}

COVID-19 is a new disease of the century caused by novel corona virus (SARS-CoV-2). The disease has its origin from Wuhan city of Hubei Province of China. The virus, belongs to RNA family and continues to spread throughout the world, causing more than $75,98,803$ people infected with COVID-19 and the number of deaths totaled to $4,23,868$ as on 12 June $2020 .{ }^{1}$ More than 3 lakh cases and 4000 deaths have been reported from India as on 12 June 2020. The disease spreads either by direct contact or through droplets generated by infected people while sneezing and coughing. The droplets ( $>5$ microns) containing virus remain alive and suspended for few hours. The incubation period of COVID-19 varies from 2 to 14 days with the median duration of 5 days. An infected individual on an average can infect 2-3 healthy individuals. Infection is acquired by either inhalation of infected droplets or touching surfaces/ fomites contaminated with the infected droplets and then touching eyes, nose and mouth. In majority of cases (85\%), the disease appears in mild to moderate form, while around $10 \%$ of cases would be severely ill and $5-6 \%$ of patients are critically ill. ${ }^{2}$

Pregnant women and neonates are generally considered to be the vulnerable groups. Pregnant women with diabetes mellitus, lung disease, renal failure and immunocompromised are more at risk. Some published case series have reported a higher number of preterm deliveries than expected (15 out of 32 deliveries were preterm. ${ }^{3,4}$ The currently available data suggests that the outcome in pregnant women and neonates during COVID-19 pandemic is good. However, it is always advisable to observe the contact and transmission based precautions by the Health Care Workers (HCW), while providing care to mother and neonate. ${ }^{3}$ Schwartz et al. studied 38 COVID positive Chinese women (confirmed by rt-PCR) in perinatal period. None of the delivered neonates had rt-PCR-confirmed SARS-CoV2 infection, suggesting that 
the virus is not identified in the placental or amniotic fluid or breast milk, ruling out maternal-fetal transmission. ${ }^{5}$ Neonatal exposure to COVID-19 infection can occur in postnatal period from COVID positive mother/family members, HCW, and visitors. On exposure, irrespective of their symptomatic or asymptomatic status, the neonates should be managed as Patients Under Investigation (PUI). During early epidemic in China, there were two reported cases of neonatal SARS-CoV-19 infection; one at day 17 of life and other one found infected at 36 hours of life following delivery. Both neonates had the history of close contact with confirmed cases, suggesting postpartum neonatal infection acquired through an infected contact., 5

The purpose of this review article is to summarize the available evidence at this moment, and to provide midwives and neonatal nurses with practical tools for managing their patients in the delivery room and NICU. The guidelines in this document are based on the currently available evidence. As new evidence accumulates, some of the recommendations may get changed. The users should use these guidelines in accordance with the latest World Health Organization (WHO), Centre for Disease Control (CDC) and national [Ministry of Health and Family Welfare (MOHFW) and Indian Council of Medical Research (ICMR)] advisories.

\section{Perinatal Management}

\section{Triaging}

All pregnant women (not in active labor) reporting to the hospital should be asked about the history of overseas travel or with exposure to a confirmed/suspected case of COVID-19, symptoms of COVID-19, coming from hot spot or on immuno-suppressants. In these instances, she should be isolated by using the guidelines for non-pregnant adults. ${ }^{8}$ In the absence of community spread, isolation at the designated facility and in the presence of community spread, isolation by home quarantine may be preferred (Figure 1). Pregnant women should be advised to maintain social distancing, use face mask and practice frequent hand hygiene.

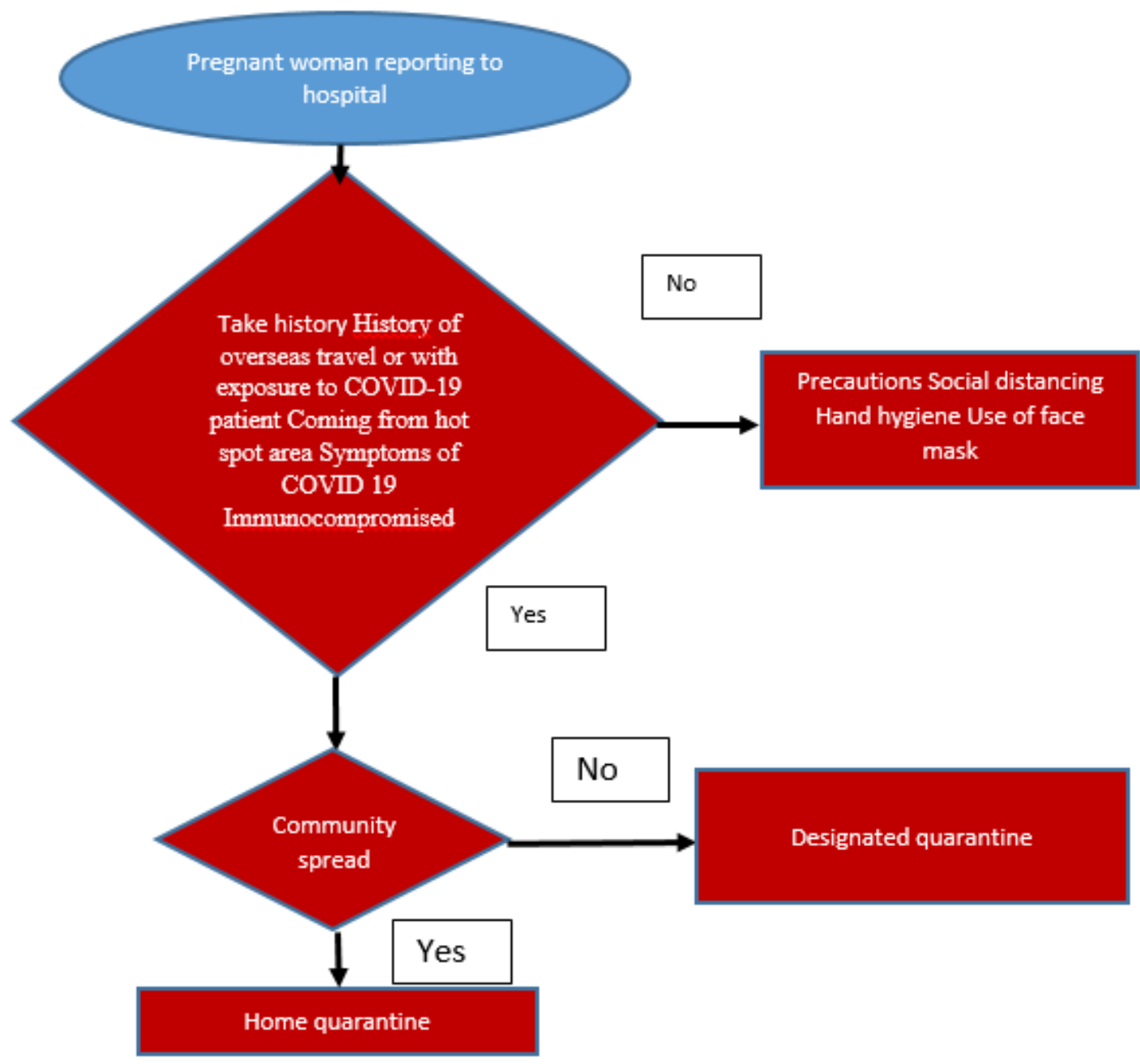

Figure I.Triaging 
In case of anticipated preterm delivery of COVID-19 positive pregnant woman, one should consider giving antenatal steroids after doing risk and benefit analysis. The use of steroids should be done carefully as it may affect maternal response to infection in COVID-19, as the use of antenatal steroids for possible premature delivery remains unknown. ${ }^{7,8}$

\section{Criteria for Offering a Laboratory Test}

The criteria for offering a laboratory test are the same for pregnant women and the non-pregnant population. ${ }^{8}$ However prior to elective delivery or caesarian section, all pregnant women having contact history with COVID-19 or coming from high risk zone should be tested for COVID-19.

\section{Management of COVID-19 Pregnant Women}

\section{Antepartum Care}

- Advise pregnant woman to attend minimum 4 visits at 12, 20, 28 and 36 weeks of gestation, unless they meet current self-isolation criteria.

- Pregnant woman who has symptoms, can be provided teleconsultation, unless symptoms are severe. Ask her to monitor the fetal kick count daily.

- $\quad$ Refer pregnant woman for antenatal ultrasound services for fetal growth surveillance, 14 days following the resolution of acute illness.

- Women should restrict attendance of antenatal clinic to themselves and a companion of choice who would stay with them throughout labor.

- Health providers should be attention to the signs of depression and domestic abuse which are on the rise during this pandemic and offer appropriate referral. ${ }^{8,9}$

\section{Intra-partum care}

- Management of COVID-19 pregnant woman in intrapartum period includes preparation of pregnant woman, preparation of the delivery room, monitoring pregnant woman and providing supportive care in intrapartum and postpartum period.

\section{a. Preparation of Pregnant Woman}

The pregnant woman should be kept in isolation room with surgical mask on for source containment for continuous monitoring. This is done mainly to protect the HCW from getting infection. She should be transferred to a separate labor room after the onset of active labor for delivery Visitors are usually restricted. However, interaction with spouse or family members can be done through digital media. A birth companion who has been counseled during antenatal period can be allowed with the woman during her hospital stay. HCW should in no way compromise on provision of respectful maternity care to woman during her hospital stay, which includes compassion, respect and dignity. ${ }^{8,9}$

\section{b. Preparation of Delivery Room and Mode of Delivery}

Follow standard universal precautions to prevent contact with body fluids. Use personal protective equipment (PPE) to prevent acquiring infection through respiratory droplets including N95 mask and face protection by a face shield, goggles, gown, and shoe covers. One should restrict the number of HCW in the delivery room.

- Arrange separate delivery room and operation theaters for management of suspected or confirmed COVID-19 mothers.

- Arrange adequate resources such as space, equipment, supplies and trained healthcare providers for delivery, caesarean section and neonatal resuscitation.

\section{Mode of Delivery}

- Obstetrician decides the mode of delivery in a pregnant woman infected with COVID-19 on the basis of obstetric assessment and physiological stability (cardiorespiratory status and oxygenation). One should remember that COVID-19 infection itself is not an indication for induction of labor or operative delivery. ${ }^{10,11}$ In fact, operative delivery can increase the duration of hospital stay and increased deployment of manpower for care of the woman. Thus potentially causing exposure of $\mathrm{HCW}$ to the virus.

\section{c. Monitoring}

Do continuous electronic fetal monitoring during labor. If facilities for the continuous electronic fetal monitoring are not available, manual monitoring by frequent auscultation of fetal heart rate should be done during the labor as indicated for a high-risk delivery.

Monitor oxygenation status of women during labor by a pulse oximeter and oxygen therapy should be titrated to maintain oxygen saturation of more than $94 \%$.

In case if pregnant woman is becoming hypoxic or exhausted, shortening of second stage of labor with instrumental delivery can be done.

Supportive and specific therapy is recommended for pregnant women with active COVID-19 infection. ${ }^{9}$ The therapy includes:

- Oxygen therapy/ respiratory support

- Fluid therapy

- Antibiotics

- Shock management

- Specific drugs in severe cases.

Drugs prescribed by the physician for the pregnant women are given as under:

- Hydroxychloroquine $200 \mathrm{mg}$ thrice a day with meals for 10 days or $400 \mathrm{mg}$ twice a day on day 1 and $400 \mathrm{mg}$ once a day for 4 days and Azithromycin 500 mg twice 
a day for 7 days (This is recommended in countries where malaria infection is high).

- Lopinavir/Ritonavir (200 mg/50 mg) - 2 tablets twice daily Or Lopinavir 400mg/Ritonavir $100 \mathrm{mg}-5 \mathrm{~mL}$ suspension twice daily.

- Duration: 14 days or for 7 days after becoming asymptomatic.

Note: The choice of specific antiviral therapy is likely to change with rapidly emerging evidence and updated national guidance should be consulted. Administer drugs only on prescription of the physician.

\section{Postpartum Care}

Postpartum care should include continuous monitoring, providing respiratory support, nutritional support and fluid therapy etc. Stable postpartum mother can be encouraged to participate in newborn care while taking all the precautions like use of mask, and hand hygiene.

\section{Newborn Care}

\section{a. Preparation of Newborn Corner}

Follow standard universal precautions to prevent contact with body fluids. Use personal protective equipment (PPE) to prevent acquiring infection through respiratory droplets including $\mathrm{N} 95$ mask and face protection by a face shield, goggles, gown, and shoe covers. One should restrict the number of HCW in newborn corner. ${ }^{8,12,13}$ The goal is to protect the neonate from getting infected with COVID-19.

- Prepare neonatal resuscitation corners located at least $2 \mathrm{~m}$ away from the delivery table.

- Arrange adequate resources such as space, equipment, supplies and trained HCW for neonatal resuscitation.

\section{b. Care at Birth}

Based on the condition of the neonate at the time of delivery, it is decided whether to provide routine care or proceed for Neonatal Resuscitation (NRP) (Figure 2).

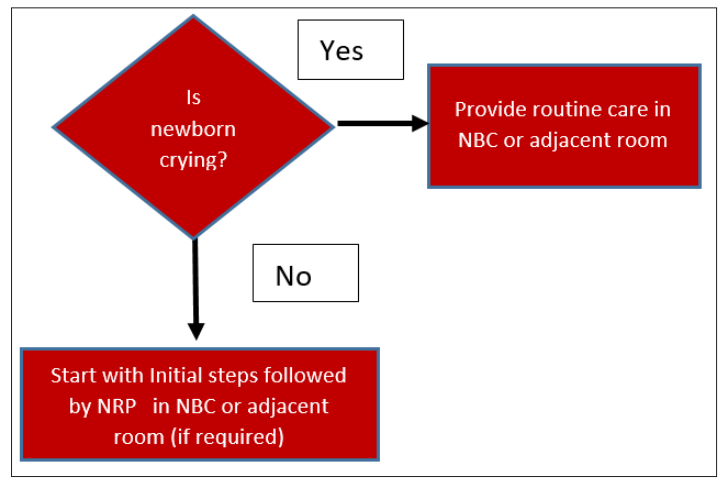

Figure 2.Assessment of neonate

- Assess neonate for breathing or crying.

- Perform early cord clamping

- Avoid early skin to skin contact
- Attend neonate following the birth in a delivery corner 2 meters away from the mother or in another room.

- It is recommended to have minimum number of health care personnel (HCP) for attending the delivery. One $\mathrm{HCP}$ is generally sufficient in normal or low risk baby, while two are needed in case of high risk delivery. Since the safety of HCP is paramount, they should be donned in PPE including N-95 mask.

- Delivery team member should hand over neonate to the resuscitation team for further management in an adjacent room.

\section{Routine Care}

Routine care is recommended for normal, low risk babies.

- Keep stable neonate exposed to stable COVID-19 infected mother in an isolation facility.

- Encourage stable mothers to initiate breast feeding within half an hour of the delivery. Ensure that no prelacteal feeds are given by the mother or family relatives.

- Make sure that mother performs hand and strict breast hygiene and wear triple layer mask at the time of feeding her neonate.

- If rooming-in is not possible due to maternal illness, the neonate should be fed expressed breast milk (EBM) of the mother by a nurse or family member who has not been in contact with the mother or other suspected/ proven case. ${ }^{12-14}$

- Bath to a stable newborn can be given at a reasonable time i.e. 24 hours after birth in order to eliminate any possible virus presence on skin of neonate. ${ }^{8}$

Note: Giving Kangaroo Mother Care (KMC) to a low birth weight (LBW) newborn is a shared decision between the $\mathrm{HCW}$ and the parents. The discussion should include risks of exposure to both the neonate and to HCW..$^{8,11}$

\section{Neonatal Resuscitation}

In case if neonate is not crying or breathing, start with initial steps and follow standard NRP guidelines. Use of selfinflating bag and mask is preferred over T-piece resuscitator, in case of need for Positive-Pressure Ventilation (PPV) in a negative air pressure room adjacent to the delivery room.

\section{NICU Admission}

Symptomatic sick neonate borne to COVID-19 infected mother is admitted in isolation facility of NICU having negative air pressure facility. Respiratory support is provided using lung protective strategy including noninvasive ventilation. Supportive care such as temperature maintenance, IV fluids, feeding and monitoring are continued.

Note: Preferably avoid NIPPV and high flow nasal cannulas. Healthcare providers should practice contact and droplet isolation and wear PPE including N95 mask while providing care to the neonate. Use of antivirals or chloroquine/ 
hydroxychloroquine (where ever applicable), systemic corticosteroids and intravenous gamma globulin are NOT recommended in symptomatic neonates with confirmed or suspected COVID-19. ${ }^{8}$

\section{Testing Protocol for Neonates}

Infants born to COVID-19 women should be tested for SARS-CoV-2 at 24 to 48 hours by doing Real-Time Polymerase Chain Reaction ( $r$ PCR) after birth. If neonate is symptomatic, collect the specimen as soon as possible. If mother is COVID-19 positive and baby's initial sample is negative, another sample should be repeated after 48 hours. Nasopharyngeal/oropharyngeal or rectal swab is considered sufficient for diagnosis. , $^{8,13}$

\section{Disinfection and Sterilization}

Disinfection of surfaces in the childbirth/neonatal care areas is done with $0.5 \%$ sodium hypochlorite solution. Sodium hypochlorite should be a freshly prepared solution.

Get floors and walls at least once per shift and after a patient is transferred out of the area.

The equipment used during child birth should be treated with multi-enzyme solution to reduce the bio-burden, followed by cleaning with soap and water solution before applying the disinfectant or sending them for autoclaving.

Ethyl alcohol (70\%) can be used to disinfect small areas between uses, especially for disinfecting the reusable dedicated equipment.

Use hydrogen peroxide (dilute $100 \mathrm{~mL}$ of $\mathrm{H}_{2} \mathrm{O}_{2} 10 \%$ $\mathrm{v} / \mathrm{v}$ solution with $900 \mathrm{~mL}$ of distilled water) for surface cleaning of incubators, open care systems, infusion pumps, weighing scales, standby equipment ventilators, monitors, phototherapy units, and shelves with a contact period of 1 hour. Use $\mathrm{H}_{2} \mathrm{O}_{2}$ only when equipment is not being used for the patient.

\section{Waste Disposal}

The clinical waste generated during the delivery of a COVID-19 woman should be properly segregated and stored in double plastic bags and labeled as COVID waste. Doffing should be done very carefully as the risk of acquiring the disease by HCW during doffing is very high. Discard the clinical waste as per the institutional guidelines.

\section{Immunization}

Immunize the neonate with suspected/proven infection, before discharge from the hospital as per existing policy. All neonates are given BCG vaccination, and ' 0 ' dose of OPV and first dose of Hepatitis B before discharge.

\section{Discharge of Neonate}

Discharge stable neonate exposed to COVID-19 and being roomed-in with her mother together at time of mothers' discharge. While, stable neonate in whom rooming-in is not possible because of sick mother, newborn be discharged from the facility by 24-48 hours of age and handed over to the family members. Sick neonates are taken care of in the isolation facility.

\section{Conclusion}

Approaches to maternal and neonatal care have been summarized considering the available evidence.

These recommendations are subjected to change as more evidence becomes available. It is also of paramount importance that HCW protect themselves while providing the best possible maternal and neonatal care.

Conflict of Interest: None

\section{References}

1. Q \& A on coronaviruses (COVID-19). Cited 2020 Jun 12. Available from: https://www.who.int/emergencies/ diseases/novel-coronavirus-2019/question-andanswers-hub/q-a-detail/q-a-coronaviruses.

2. CDC. Interim Considerations for infection prevention and control of coronavirus disease 2019 (COVID-19) in inpatient obstetric healthcare settings. 2020. Available from: https://www.cdc.gov/coronavirus/2019-ncov/ hcp/inpatient-obstetric-healthcareguidance.html.

3. Chen H, Guo J, Wang C et al. Clinical characteristics and intrauterine vertical transmission potential of COVID-19 infection in nine pregnant women: a retrospective review of medical records. Lancet 2020; 395(10226): 809-815.

4. Schwartz DA, Graham AL. Potential maternal and infant outcomes from (Wuhan) Coronavirus 2019nCoV infecting pregnant women: Lessons from SARS, MERS, and Other human coronavirus infections. Viruses 2020; 12(2): 194.

5. Mimouni F, Lakshminrusimha S, Pearlman SA et al. Perinatal aspects on the covid-19 pandemic: a practical resource for perinatal-neonatal specialists. Journal of Perinatology Cited 2020 Jun 12. Available from: https:// www.nature.com/articles/s41372-020-0665-6.

6. Zhang $L$, Jiang $\mathrm{Y}, \mathrm{Wei} \mathrm{M}$ et al. Analysis of the pregnancy outcomes in pregnant women with COVID-19 in Hubei Province. Zhonghua Fu Chan Ke Za Zhi 2020; 55(0): E009.

7. Klein K, McClure EM, Colaci D et al. The antenatal corticosteroids trial (ACT): a secondary analysis to explore site differences in a multi-country trial. Reprod Health 2016; 13(01): 64.

8. Chawla D, Chirla D, Dalwai S et al. Perinatal-neonatal management of COVID-19 infection - Guidelines of the Federation of Obstetric and Gynecological Societies of India (FOGSI), National Neonatology Forum of India (NNF), and Indian Academy of Pediatrics (IAP). Indian 
Pediatr., 2020.

9. Guidance_for_Management_of_Pregnant_Women_ in_COVID19_Pandemic_12042020.pdf. Cited 2020 Jun 12. Available from: https://www.icmr.gov.in/pdf/covid/ techdoc/Guidance_for_Management_of_Pregnant_ Women_in_COVID19_Pandemic_12042020.pdf.

10. Coronavirus (COVID-19) infection in pregnancy and newborn: Guidelines, reviews, statements, recommendations, standards. Retrieved June 12, 2020. Available from: https://www.gfmer.ch/Guidelines/ Maternal_neonatal_infections/Coronavirus.htm.

11. Europe PMC. Cited 2020 Jun 12. Available from: https:// europepmc.org/article/med/32238615.

12. Mullins E, Evans D, Viner RM et al. Coronavirus in pregnancy and delivery: rapid review. Ultrasound Obstet Gynecol 2020.

13. Ma X, Zhu J, Du L. Neonatal management during the Coronavirus Disease (COVID-19) outbreak: The Chinese experience. NeoReviews 2020; 21(5): e293-7.

14. AAP guidelines for neonatal care of infants born to COVID-19 mothers. Retrieved June 12, 2020. Available from: https://www.m3india.in/contents/ editor_pick/138151/aap-guidelines-for-neonatal-caremanaging-infants. 